\title{
Pose-Based SLAM with Probabilistic Scan Matching Algorithm using a Mechanical Scanned Imaging Sonar
}

\author{
Angelos Mallios, Pere Ridao \\ Emili Hernández and David Ribas \\ Department of Computer Engineering \\ University of Girona \\ 17071 Girona, Spain \\ Email: \{amallios,pere,emilihb,dribas\}@eia.udg.edu
}

\author{
Francesco Maurelli and Yvan Petillot \\ Earl Mountbatten Building \\ Heriot-Watt University \\ EH14 4AS Edinburgh, UK \\ Email: f.maurelli@ieee.org, y.r.petillot@hw.ac.uk
}

\begin{abstract}
This paper proposes a pose-based algorithm to solve the full SLAM problem for an Autonomous Underwater Vehicle (AUV), navigating in an unknown and possibly unstructured environment. The technique incorporate probabilistic scan matching with range scans gathered from a Mechanical Scanning Imaging Sonar (MSIS) and the robot dead-reckoning displacements estimated from a Doppler Velocity Log (DVL) and a Motion Reference Unit (MRU). The proposed method utilizes two Extended Kalman Filters (EKF). The first, estimates the local path travelled by the robot while grabbing the scan as well as its uncertainty and provides position estimates for correcting the distortions that the vehicle motion produces in the acoustic images. The second is an augment state EKF that estimates and keeps the registered scans poses. The raw data from the sensors are processed and fused in-line. No priory structural information or initial pose are considered. The algorithm has been tested on an AUV guided along a $600 \mathrm{~m}$ path within a marina environment, showing the viability of the proposed approach.
\end{abstract}

\section{INTRODUCTION}

In spite of the recent advances in unmanned underwater vehicles (UUV) navigation techniques, robustly solving their localization in unstructured and unconstrained areas is still a challenging problem. The last decades, a number of studies in mobile robotics had developed techniques to address the localization problem with very promising results. In particular, the so-called Simultaneous Localization and Mapping (SLAM) techniques have been broadly and successfully applied to indoor and outdoor environments [1]. Hence, it is of interest to study how to adapt these techniques for their use in hostile underwater environments. Underwater SLAM for UUVs can be classified, from the sensors vantage point, in two main categories: vision and sonar based SLAM. Underwater vision, in the best conditions, is restricted to few meters from the vehicle but its normaly rich in information and has very good resolution. On the other hand, sonar has the ability of long distance penetration, even in turbulent waters, but with the downside of sparsely and noisy readings. In both cases, with the physical properties of the media, the unstructured environment and the poor quality of the data gathered with a conventional sensor suite, underwater SLAM becomes real challenging. For this reason, when compared with land robotics, very few SLAM algorithms working with real data have been reported in the literature [2].
This paper is a contribution in this area, proposing a pose-based algorithm to solve the full SLAM problem of an AUV navigating in an unknown and possibly unstructured environment. A DVL and a low cost gyrocompass are used for dead reckoning while a mechanical scanning imaging sonar (MSIS) is used for sensing the environment. Scan matching is a technique that can be used to estimate the vehicle displacement using successive range scans. Many applications in robotics like mapping, localization or tracking use this technique to estimate the robot's relative displacement [3], [4] (to mention some but a few). Scan Matching estimates the robot relative displacement between two configurations, by maximizing the overlap between the range scans normally gathered with a laser or a sonar sensor. The existing scan matching techniques can be divided in two groups depending on if they use high-level entities like lines or planes or otherwise they rely on the raw scan. On one hand, it is possible to assume the existence of polygonal structures in structured environments as is supposed in [5] [6], or even in some underwater applications [7]. However, extracting simple polygons for representing the environment is not always possible, particularly in unstructured scenarios which are the most common in underwater robotics. On the other hand, there is a second type of algorithms that work with raw data from the scanner to solve the matching. Usually, these techniques are based on a two step iterative process which is repeated till convergence. The sensor displacements are computed by approximating the solution to the best overlap between two scans by looking for the closest point for each single data of the scan. After that, a minimization process to estimate the solution is done. The process is repeated until convergence.

The most popular technique is the ICP (Iterative Closest Point) algorithm [8] which has been modified in different ways [9]. However, most of these algorithms do not take into account the sensor or the displacement uncertainties which are very important, especially when sonar sensors are used. The probabilistic Iterative Correspondence method (pIC), proposed in [10], explicitly deals with those uncertainties to decide which points in the reference scan are statistically compatible with a certain point of the new scan. A probabilistic weight average is used then to compute a virtual association point for the matching during the minimization [11]. Although the 
method is suitable for laser data, the same authors have noted that for sparse noisy data like sonar, better results can be achieved using the ICNN data association algorithm instead of using the virtual point ( $\mathrm{spIC}$ ). A reduced version of the spIC algorithm is used in this paper, being extended with the MSISpIC algorithm proposed in [12], to dial with data gathered by an AUV utilizing MSIS. Hence, an EKF using a constant velocity model with acceleration noise, updated with velocity and attitude measurements obtained from a DVL and a MRU respectively, is used to estimate the trajectory followed by the robot along the scan. This trajectory is used to remove the motion induced distortion of the acoustic image as well as to predict the uncertainty of the range scans prior to register them through the spIC algorithm.

In this paper we extend the MSISpIC algorithm in the pose-based SLAM framework. Now, each new pose of a scan is maintained in a second augment state EKF (ASEKF) and is compared with previous scans that are in the nearby area. If there is enough data overlapping, a new scan match will put a constrain between the poses updating the ASEKF. These constrains help to identify and close the loops which correct the entire previously trajectory, bounding the drift. The proposed method has been tested with a real world dataset, including DGPS for ground truth, acquired during a survey mission in an abandoned marina located in the Girona coast. The results show substantial improvements in trajectory correction and map reconstruction.

The paper is structured as follows. In section II the probabilistic scan matching algorithm is described. Section III details the MSISpIC to be used in our SLAM algorithm which is described in section IV. Section V reports the experimental results before conclusions and future work.

\section{PROBABILISTIC SCAN MATCHING}

The geometric representations of the scan in the conventional ICP algorithm do not model the uncertainty of the sensor measurements. Correspondences between two scans are chosen based on the closest-point rule normally using the Euclidean distance. As pointed out in [10], this distance do not take into account that the points in the new scan, which are far from the sensor, could be far from their correspondents in the previous scan. On the other hand, if the scan data is very noisy, two statistically compatible points could appear far enough, in terms of the Euclidean distance. Both situations might prevent a possible association or even generate a wrong one. The spIC algorithm proposed in [11] is a statistical extension of the ICP algorithm where the relative displacement $\mathbf{q}$ as well as the observed points in both scans $\mathbf{r}_{\mathbf{i}}$ and $\mathbf{n}_{\mathbf{i}}$, are modeled as random Gaussian variables (r.g.v.). For a better understanding the algorithm is reproduced in Algorithm 1. The inputs are the reference scan $S_{r e f}$ with points $\mathbf{r}_{\mathbf{i}}(i=1 . . n)$, the new scan $S_{n e w}$ with points $\mathbf{n}_{\mathbf{j}}(j=1 . . m)$ and the initial relative displacement estimation $\hat{\mathbf{q}}$ with its covariance $\mathbf{P}_{\mathbf{q}}$. The following procedure is iteratively executed until convergence. First, the points of the new scan $\left(\mathbf{n}_{\mathbf{j}}\right)$ are compounded with the robot displacement $\left(\mathbf{q}_{\mathbf{k}}\right)$. The result $\left(\mathbf{c}_{\mathbf{j}}\right)$, are the points of the new scan referenced to the reference frame. Then, for each point $\left(\mathbf{c}_{\mathbf{j}}\right)$, its association point $\left(\mathbf{a}_{\mathbf{j}}\right)$ is computed using individual compatibility nearest neighbor (ICNN) algorithm. Note that $\mathbf{q} \equiv N\left(\hat{\mathbf{q}}, \mathbf{P}_{\mathbf{q}}\right), \mathbf{n}_{\mathbf{j}} \equiv N\left(\hat{\mathbf{n}}_{\mathbf{j}}, \mathbf{P}_{\mathbf{n}_{\mathbf{j}}}\right)$ and $\mathbf{r}_{\mathbf{i}} \equiv N\left(\hat{\mathbf{r}}_{\mathbf{i}}, \mathbf{P}_{\mathbf{r}_{\mathbf{i}}}\right)$ are r.g.v. which describes the error of the $\left\{\mathbf{r}_{\mathbf{i}}, \mathbf{c}_{\mathbf{j}}\right\}$ pairing:

$$
\begin{aligned}
\mathbf{e}_{\mathbf{j}} & =\mathbf{a}_{\mathbf{i}}-\mathbf{q}_{\mathbf{k}} \oplus \mathbf{n}_{\mathbf{j}} \\
\mathbf{e}_{\mathbf{j}} & \cong N\left(\hat{\mathbf{a}}_{\mathbf{i}}-\hat{\mathbf{q}_{\mathbf{k}}} \oplus \hat{\mathbf{n}}_{\mathbf{j}}, \mathbf{P}_{\mathbf{e}_{\mathbf{j}}}\right) \\
\mathbf{P}_{\mathbf{e}_{\mathbf{j}}} & =\mathbf{P}_{\mathbf{a}_{\mathbf{i}}}+\mathbf{J}_{\mathbf{q}} \mathbf{P}_{\mathbf{q}} \mathbf{J}_{\mathbf{q}}{ }^{T}+\mathbf{J}_{\mathbf{n}} \mathbf{P}_{\mathbf{n}_{\mathbf{j}}} \mathbf{J}_{\mathbf{n}}{ }^{T}
\end{aligned}
$$

with

$$
\mathbf{J}_{\mathbf{q}}=\left.\frac{\partial \mathbf{a}_{\mathbf{i}}-\mathbf{q} \oplus \mathbf{n}_{\mathbf{j}}}{\partial \mathbf{q}}\right|_{\hat{\mathbf{q}}}, \mathbf{J}_{\mathbf{n}}=\left.\frac{\partial \mathbf{a}_{\mathbf{i}}-\mathbf{q} \oplus \mathbf{n}_{\mathbf{j}}}{\partial \mathbf{n}_{\mathbf{j}}}\right|_{\hat{\mathbf{n}}_{\mathbf{j}}}
$$

$\mathbf{P}_{\mathbf{e}_{\mathbf{j}}}$ is the uncertainty of the matching error $\left(\mathbf{a}_{\mathbf{j}}-\mathbf{c}_{\mathbf{j}}\right)$ which is used to estimate the displacement $\hat{\mathbf{q}}_{\min }$ through the minimization of the squares error of the Mahalanobis Distance [13] between $\mathbf{a}_{\mathbf{j}}$ and $\mathbf{c}_{\mathbf{j}}$. This is done using Least Squares minimization method. If there is convergence, the function returns, otherwise another iteration is required.

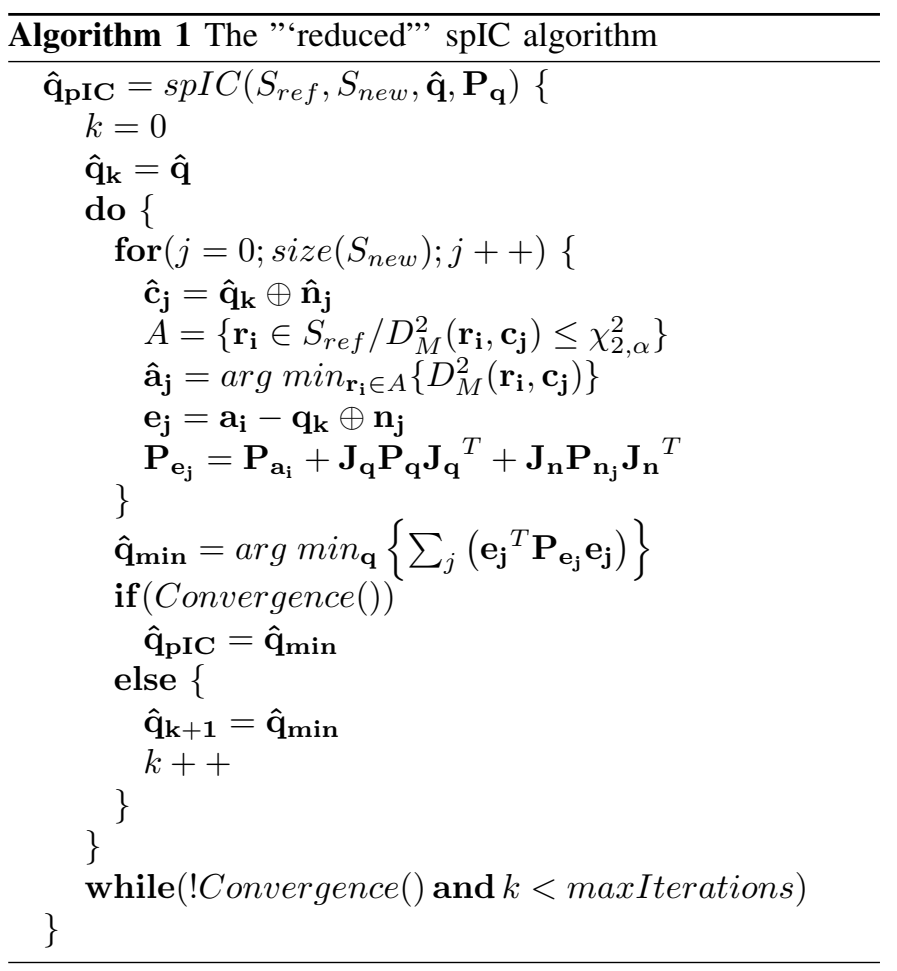

\section{MSISPIC ALGORITHM}

Scan matching techniques are conceived to accept as input parameters two range scans with a rough displacement estimation between them. Most of the algorithms use laser range finders which gather scans almost instantaneously. However, for the underwater environment, commercially available scan sensors are based on acoustics. Most of these sensors have a mechanical head that rotates at fixed angular steps. At each step, a beam is emitted and received a posteriori, measuring ranges to the obstacles found across its trajectory. Thus, getting 
a complete scan that lasts few seconds while the vehicle is moving, generating deformed acoustic images. Therefore, a correction taking into account the robot pose when the beam was grabbed is necessary. This is part of the MSISpIC algorithm (see Algorithm 2). It forms a scan, corrected from the vehicle's motion distortion, through the ScanGrabbing algorithm (detailed below). ScanGrabbing uses an EKF with a constant velocity model and acceleration noise for the prediction step. Then, it updates with velocity and attitude measurements obtained from a DVL and a MRU respectively, in order to estimate the trajectory followed by the robot along the scan. This trajectory is used to remove the motion induced distortion of the acoustic image as well as to predict the uncertainty of the range scans prior to register them. After the corrected scan has formed, MSISpIC grabs two scans and registers them using the spIC algorithm. It is worth noting that the pIC takes as input two consecutive scans $\left(S_{\text {new }}\right.$ and $\left.S_{r e f}\right)$ and its relative displacement which coincides with the position occupied by the robot at the end of the first scan ( $\left.\hat{\mathrm{q}}_{\mathrm{ref}}\right)$. The output is an improved estimation of the robot displacement ( $\left.\hat{\mathbf{q}}_{\text {new }}\right)$ which compounding allows to track the global robot position. ScanGrabbing algorithm, consist of three

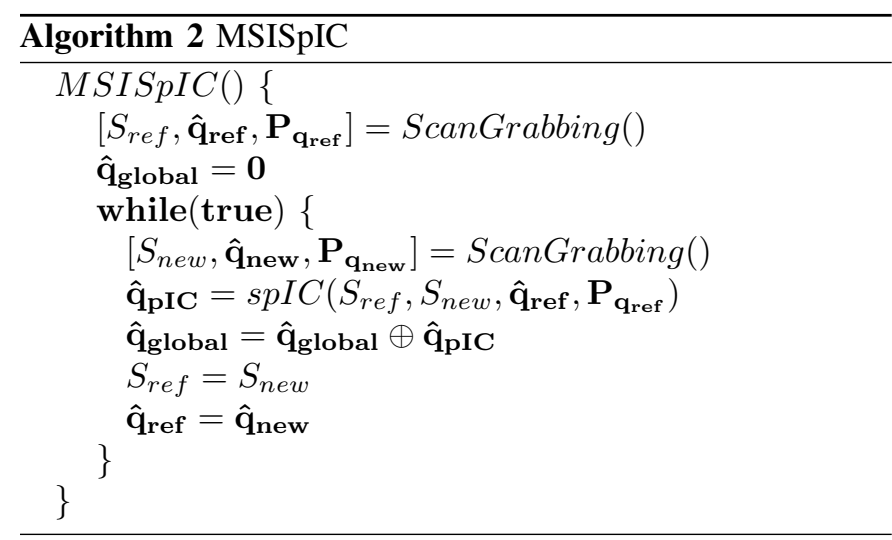

major parts: Beam segmentation, Relative vehicle localization and Scan forming.

\section{A. Beam segmentation and range detection}

The MSIS returns a polar acoustic image composed of beams. Each beam has a particular bearing angle value and a set of intensity measurements. The angle corresponds to the orientation of the sensor head when the beam was emitted. The acoustic linear image corresponding to one beam is returned as an array acoustic intensities detected at a certain distance. The beam is then segmented using a predefined threshold to compute the intensity peaks. Due to the noisy nature of the acoustic data, a minimum distance between peaks criteria is also applied. Hence, positions finally considered are those corresponding to high intensity values above the threshold with a minimum distance between each other.

\section{B. Relative vehicle localization}

The spIC algorithm needs a complete scan to be registered with the previous one in order to estimate the robot's dis-

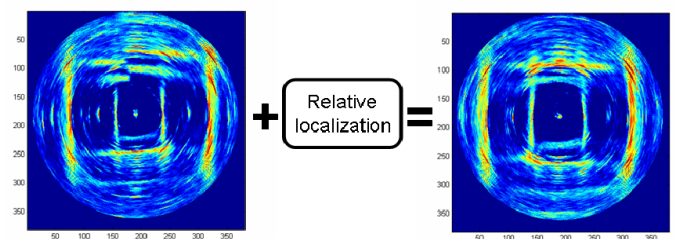

Fig. 1. The distortion produced by the displacement of the robot while acquiring data can be corrected with the relative displacement.

placement. Since MSIS needs a considerable period of time to obtain a complete scan, if the robot does not remain static, the robot's motion induces a distortion in the acoustic image (Fig. 1). To deal with this problem it is necessary to know the robot's pose at the beam reception time. Hence, it is possible to define an initial coordinate system $I$ to reference all the range measurements belonging to the same scan. In our case, this initial frame is fixed at the robot pose where the first beam of the current scan was read. The localization system used in this work is a slight modification of the navigation system described in [14]. In this system, a Xsense MTi MRU provides heading measurements and a SonTek Argonaut DVL unit which includes 2 inclinometers and a depth sensor is used to estimate the robot's pose during the scan (navigation problem). MSIS beams are read at $30 \mathrm{~Hz}$ while DVL and MRU readings arrive asynchronously at a frequency of 1.5 $\mathrm{Hz}$ and $10 \mathrm{~Hz}$ respectively. An EKF is used to estimate the robot's 6DOF pose whenever a sonar beam is read. DVL and MRU readings are used asynchronously to update the filter. To reduce the noise inherent to the DVL measurements, a simple 6DOF constant velocity kinematics model is used.

The information of the system at step $k$ is stored in the state vector $\mathbf{x}_{k}$ with estimated mean $\hat{\mathbf{x}}_{k}$ and covariance $\mathbf{P}_{k}$ :

$$
\hat{\mathbf{x}}_{k}=\left[\hat{\eta}^{B}, \hat{\nu}^{R}\right]^{T} \mathbf{P}_{k}=E\left[\left(\mathbf{x}_{k}-\hat{\mathbf{x}}_{k}\right)\left(\mathbf{x}_{k}-\hat{\mathbf{x}}_{k}\right)^{T}\right]
$$

with:

$$
\eta^{B}=[x, y, z, \phi, \theta, \psi]^{T} ; \quad \nu^{R}=[u, v, w, p, q, r]^{T}
$$

where, as defined in [15], $\eta^{B}$ is the position and attitude vector referenced to a base frame $B$, and $\nu^{R}$ is the linear and angular velocity vector referenced to the robot's coordinate frame $R$. The coordinate frame $B$ is chosen coincident with I but oriented to the north, hence the compass measurements can be integrated straight forward.

The vehicle's movement prediction is performed using the 6DOF kinematic model:

$$
\mathbf{x}_{k}=f\left(\mathbf{x}_{k-1}\right)=\left[\begin{array}{l}
\eta_{k}^{B} \\
\nu_{k}^{R}
\end{array}\right]=\left[\begin{array}{c}
\eta_{k-1}^{B}+J\left(\eta_{k-1}^{B}\right) \nu_{k-1}^{R} T \\
\nu_{k-1}^{R}
\end{array}\right]
$$

$$
J(\eta)=\left[\begin{array}{cccccc}
c \psi c \theta & c \psi s \theta s \phi-s \psi c \phi & c \psi s \theta c \phi+s \psi s \phi & 0 & 0 & 0 \\
s \psi c \theta & s \phi s \psi s \theta+c \psi c \phi & s \psi s \theta c \phi-s \phi c \psi & 0 & 0 & 0 \\
-s \theta & c \theta s \phi & c \theta c \phi & 0 & 0 & 0 \\
0 & 0 & 0 & 1 & s \phi t \theta & c \phi t \theta \\
0 & 0 & 0 & 0 & c \phi & -s \phi \\
0 & 0 & 0 & 0 & s \phi / c \theta & c \phi / c \theta
\end{array}\right]
$$


Although in this model the velocity is considered to be constant, in order to allow for slight changes, a velocity perturbation modeled as the integral of a stationary white noise $v_{k}$ is introduced. The covariance matrix $\mathbf{Q}_{\mathrm{k}}$ of this acceleration noise is diagonal and in the order of magnitude of the maximum acceleration increment that the robot may experience over a sample period.

$$
\begin{gathered}
\nu_{k}^{R}=\hat{\nu}_{k}^{R}+v_{k} T \\
E\left[v_{k}\right]=0 ; \quad E\left[v_{k} v_{j}^{T}\right]=\delta_{k_{j}} \mathbf{Q}
\end{gathered}
$$

Hence, $v_{k}$ is an acceleration noise which is integrated and is added in velocity ( 8 ), being nonlinearly propagated to the position. Finally, the model prediction and update is carried out as detailed below:

1) Prediction: The estimate of the state is obtained as:

$$
\hat{\mathbf{x}}_{k}=f\left(\hat{\mathbf{x}}_{k-1}\right)
$$

and its covariance matrix as:

$$
\mathbf{P}_{k}=\mathbf{F}_{k} \mathbf{P}_{k-1} \mathbf{F}_{k}^{T}+\mathbf{G}_{k} \mathbf{Q}_{k} \mathbf{G}_{k}^{T}
$$

where $\mathbf{F}_{k}$ and $\mathbf{G}_{k}$ are the Jacobian matrices of partial derivatives of the non-linear model function $f$ with respect to the state $\mathbf{x}_{R, k}^{B}$ and the noise $v_{k}$, respectively.

2) Update using DVL measurements: The model prediction is updated by the standard Kalman filter equations each time a new DVL measurement arrives:

$$
\mathbf{z}_{D V L, k}=\left[u_{b}, v_{b}, w_{b}, u_{w}, v_{w}, w_{w}, \phi_{i}, \theta_{i}, \psi_{c}, z_{\text {depth }}\right]^{T}
$$

where subindex $b$ stands for bottom tracking velocity, $w$ for through water velocity, $i$ for inclinometers and $c$ represents the compass. The measurement model is:

$$
\begin{aligned}
& \mathbf{z}_{D V L, k}=\mathbf{H}_{D V L, k} \mathbf{x}_{k \mid k-1}+w_{k}
\end{aligned}
$$

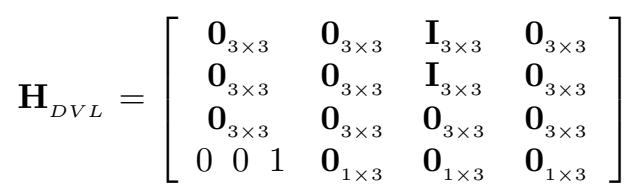

where $w_{k}$ (measurement noise) is a zero-mean white noise:

$$
E\left[w_{k}\right]=0 ; \quad E\left[w_{k} w_{j}^{T}\right]=\delta_{k_{j}} \mathbf{R}_{D V L, k}
$$

Since the DVL sensor provides a status measurement for bottom tracking and water velocity, depending on the quality of the measurements, different versions of the $\mathbf{H}$ matrix are used to fuse one (removing row 2), the other (removing row 1 ), or both readings (using the full matrix).

3) Update using MRU measurements: Whenever a new attitude measurement is available from the MRU sensor, the model prediction is updated using the standard Kalman filter equations:

$$
\begin{aligned}
\mathbf{z}_{M R U, k}=[\phi, \theta, \psi]^{T} & , \mathbf{z}_{M R U, k}=\mathbf{H}_{M R U, k} \mathbf{x}_{k \mid k-1}+w_{k} \\
\mathbf{H}_{M R U} & =\left[\begin{array}{lll}
\mathbf{0}_{3 \times 3} & \mathbf{I}_{3 \times 3} & \mathbf{0}_{3 \times 6}
\end{array}\right]
\end{aligned}
$$

where $w_{k}$ (measurement noise) is a zero-mean white noise:

$$
E\left[w_{k}\right]=0 ; \quad E\left[w_{k}, w_{j}^{T}\right]=\delta_{k_{j}} \mathbf{R}_{M R U, k}
$$

\section{Scan forming}

The navigation system presented above is able to estimate the robot's pose, but the uncertainty will grow without limit due to its dead-reckoning nature. Moreover, we are only interested in the robot's relative position (and uncertainty) with respect to the beginning of the scan $(I)$. Hence a slight modification to the filter is introduced making a reset in position (setting $x, y, z$ to 0 in the vector state) whenever a new scan is started. Therefore, while the filter is working, the estimated position is always relative to the position where the first beam of the scan was gathered $(I)$. Note that it is important to keep the $\psi$ value (it is not reset) because it represents an absolute angle with respect to the magnetic north and a reset would mean an unreal high rotation during the scan. The same thing happens with $\phi$ and $\theta$. Since we are only interested in the uncertainty accumulated during the scan, the reset process also affects the $x, y$, and $z$ terms of the covariance matrix $\mathbf{P}$. Now, the modified filter provides the robot's relative position where the beams where gathered including its uncertainty accumulated during the scan. Hence, using a similar procedure than in [16], it is possible to reference all the ranges computed from the beams to the initial frame $I$, removing the distortion induced by the robot's motion.

\section{SLAM ALGORITHM}

Our pose-based SLAM algorithm uses an augment state EKF (ASEKF) for the scans poses estimation. In this implementation of the stochastic map, the estimate of the positions of the vehicle at the end of each full scan $\left\{\mathbf{x}_{1} \ldots \mathbf{x}_{n}\right\}$ are stored in the state vector $\hat{\mathbf{x}}$.

$$
\hat{\mathbf{x}}_{k}=\left[\begin{array}{lll}
\hat{\mathbf{x}}_{1_{k}} & \ldots & \hat{\mathbf{x}}_{n_{k}}
\end{array}\right]^{T} .
$$

The covariance matrix for this state is defined as:

$$
\mathbf{P}_{\mathbf{k}}=E\left(\left[\mathbf{x}_{\mathbf{k}}-\hat{\mathbf{x}}_{\mathbf{k}}\right]\left[\mathbf{x}_{\mathbf{k}}-\hat{\mathbf{x}}_{\mathbf{k}}\right]^{T} \mid Z_{k}\right) .
$$

All the elements on the state vector are represented in the map reference frame B. Although this reference frame can be defined arbitrarily, we have chosen to place its origin on the initial position of the vehicle and to orient it to the north. Hence, the compass measurements can be straight forward integrated for vehicle's heading estimation.

\section{A. Prediction}

The pose state $\mathbf{x}_{i}$ is represented as:

$$
\mathbf{x}_{i}=\left[\begin{array}{lll}
x & y & \psi
\end{array}\right]^{T}
$$

where, $x, y$ and $\psi$ is the position and orientation vector of the vehicle. The motion model used for the prediction step is based on the compounding [17] of the previous scan pose represented in B-frame with the robot displacement ( $\hat{\mathrm{q}}_{\mathrm{ref}}$ ) returned by the 
MSISpIC scan matching algorithm:

$$
\hat{\mathbf{x}}_{\mathbf{k}}=\left[\begin{array}{c}
\hat{\mathbf{x}}_{m_{k}} \\
\hat{\mathbf{x}}_{n_{k}} \\
\vdots \\
\hat{\mathbf{x}}_{i_{k}} \\
\vdots \\
\hat{\mathbf{x}}_{1_{k}}
\end{array}\right]=\left[\begin{array}{c}
\hat{\mathbf{x}}_{n_{k}} \oplus \hat{\mathbf{q}}_{\mathbf{n e w}_{\mathbf{k}}} \\
\hat{\mathbf{x}}_{n_{k}} \\
\vdots \\
\hat{\mathbf{x}}_{i_{k}} \\
\vdots \\
\hat{\mathbf{x}}_{1_{k}}
\end{array}\right]
$$

With the new pose uncertainty represented as $\mathbf{P}_{m}$, the whole state uncertainty is updated as:

$$
\begin{gathered}
\mathbf{P}_{\mathbf{k}}=\mathbf{F P}_{\mathbf{k}} \mathbf{F}^{T}+\mathbf{G P}_{m_{k}} \mathbf{G}^{T} \\
\mathbf{F}=\left[\begin{array}{cccc} 
& & & \\
\mathbf{J}_{1 \oplus} & 0 & \ldots & 0 \\
0 & \mathbf{I} & \ldots & 0 \\
\vdots & \vdots & \ldots & \vdots \\
0 & 0 & \ldots & \mathbf{I}
\end{array}\right] \quad \mathbf{G}=\left[\begin{array}{c}
\mathbf{J}_{2 \oplus} \\
0 \\
\vdots \\
0
\end{array}\right]
\end{gathered}
$$

where $\mathbf{J}_{1 \oplus}$ and $\mathbf{J}_{2 \oplus}$ are the Jacobian matrices of the compounding transformation. In our case, since all the frames are oriented to the north, the compounding function becomes the linear vector addition.

\section{B. Loop closing candidates}

Each new pose of a scan is compared against the previous scans that are in the nearby area and if there is enough data overlapping, a new scan match will put a constrain between the poses, updating the ASEKF. These constrains help to identify and close the loops which correct the whole trajectory, bounding the drift. For this experiment, we set a threshold of 10 meters for the maximum range of candidates. To perform a scan matching, the spIC algorithm, as we have seen, needs two scans ( $S_{\text {new }}$ and $S_{\text {ref }}$ ) and the relative robot displacement $\left(\hat{\mathbf{q}}_{\mathrm{rel}}, \mathbf{P}_{\mathbf{q}_{\text {new }}}\right)$. If the matched scans are consecutives then $\mathbf{q}_{\mathrm{rel}}=\mathbf{q}_{\text {new }}$. In a loop closure situation the scans are placed at different points along the trajectory. To find the displacement for non consecutive scans, a tail-to-tail transformation [17] is applied:

$$
\begin{gathered}
\mathbf{q}_{\text {rel }}=\ominus \mathbf{x}_{\mathbf{i}} \oplus \mathbf{x}_{\mathbf{m}} \\
\mathbf{P}_{\mathbf{q}_{\text {rel }}}=\mathbf{J}_{1 \oplus} \mathbf{J}_{\ominus} \mathbf{P}_{i i} \mathbf{J}_{\ominus}^{T} \mathbf{J}_{1 \oplus}^{T}+\mathbf{J}_{2 \oplus} \mathbf{P}_{m m} \mathbf{J}_{2 \oplus}^{T}+\ldots \\
\ldots \mathbf{J}_{1 \oplus} \mathbf{J}_{\ominus} \mathbf{P}_{i k} \mathbf{J}_{2 \oplus}^{T}+\mathbf{J}_{2 \oplus} \mathbf{P}_{k i} \mathbf{J}_{\ominus}^{T} \mathbf{J}_{1 \oplus}^{T}
\end{gathered}
$$

where $\mathbf{J}_{1 \oplus}, \mathbf{J}_{2 \oplus}$ and $\mathbf{J}_{\ominus}$ are the Jacobian matrices of the compounding and inversion transformations.

Now, the spIC scan matching algorithm can be applied:

$$
\hat{\mathbf{q}}_{\mathbf{p I C}}=\operatorname{spIC}\left(S_{r e f}, S_{n e w}, \hat{\mathbf{q}}_{\mathrm{rel}}, \mathbf{P}_{\mathbf{q}_{\mathrm{rel}}}\right)
$$

The problem with the spIC algorithm is that it does not provide an estimation of the uncertainty of the displacement. Thus, the observation noise is assumed to be a r.g.v. with zero mean and fixed covariance:

$$
\begin{aligned}
\mathbf{R}_{\mathbf{q}} & =\operatorname{diag}\left\{{\sigma_{\mathbf{x q}}}^{2},{\sigma_{\mathbf{y q}}}^{2},{\sigma_{\mathbf{x} \psi}}^{2}\right\} \\
& =\operatorname{diag}\left\{1.5_{m}^{2}, 1.5_{m}^{2}, 1_{\text {deg }}^{2}\right\}
\end{aligned}
$$

\section{State Update}

The measurement to be used for the update is obtained as:

$$
\mathbf{z}=\hat{\mathbf{x}}_{i} \oplus \hat{\mathbf{q}}_{\mathbf{p I C}}
$$

with measurement uncertainty:

$$
\mathbf{R}_{\mathbf{z}}=\mathbf{J}_{1 \oplus} \mathbf{P}_{i i} \mathbf{J}_{1 \oplus}^{T}+\mathbf{J}_{2 \oplus} \mathbf{R}_{\mathbf{q}} \mathbf{J}_{2 \oplus}^{T}
$$

where again the $\mathbf{J}_{1 \oplus}$ and $\mathbf{J}_{2 \oplus}$ are the Jacobian matrices of the compounding transformation. Then, a linear measurement model is used:

$$
\hat{\mathbf{z}}=\mathbf{H}(\hat{\mathbf{x}})=\hat{\mathbf{x}}_{\mathbf{m}}
$$

and the $\mathbf{H}$ matrix in the measurement equation ( 30) which relates the state to the measurement $\mathbf{z}$, is:

$$
\mathbf{H}=\left[\begin{array}{llll}
\mathbf{I}_{3 x 3} & 0 & \ldots & 0
\end{array}\right]
$$

Now, an update of the stochastic map can be performed with the standard Kalman filter equations.

\section{EXPERIMENTAL RESULTS}

The method described in this paper has been used with a dataset obtained in an abandoned marina located in Sant Pere Pescador, on the Catalan coast [18] [16]. This dataset is useful to test if an algorithm is capable to register the limited information provided by each scan in a large underwater environment. It is in a structure environment but our algorithm does not take into account any structural information neither features and with the current sensor suit, can been used wherever there is enough vertical information. The survey mission was carried out using ICTINEU ${ }^{\mathrm{AUV}}$ [7] traveling along a $600 \mathrm{~m}$ path. The MSIS was configured to scan the whole $360^{\circ}$ sector and it was set to fire up to a $50 \mathrm{~m}$ range with a $0.1 \mathrm{~m}$ resolution and a $1.8^{\circ}$ angular step. Dead-reckoning was computed using the velocity reading coming from the DVL and the heading data obtained from the MRU sensor, both merged using the described EKF. Standard deviation for the MSIS sensor was set as it is specified by the manufacturer, $0.1 \mathrm{~m}$ in range and $1.8^{\circ}$ in angular measurements. Fig. 2.a shows the trajectory and the map estimated using the dead-reckoning method. Fig. 2.b shows the trajectory and the map estimated with our SLAM algorithm. In these figures, the estimated trajectory is plotted on an ortophotomap together with the GPS ground truth for comparison. It can be appreciated that the dead-reckoning estimated trajectory suffers from an important drift which is drastically reduced when our algorithm is used.

In Fig. 2.b it can be appreciated that the mapped size of the polygonal channel is smaller than the actual size whilst in the long, almost horizontal, water channel some times is smaller and some longer. This problem arises because during part of the trajectory, the robot traverses an area where the scan only observes one or two walls parallel to the robot path, being able to correct the lateral displacement but still drifting in the forward direction. 


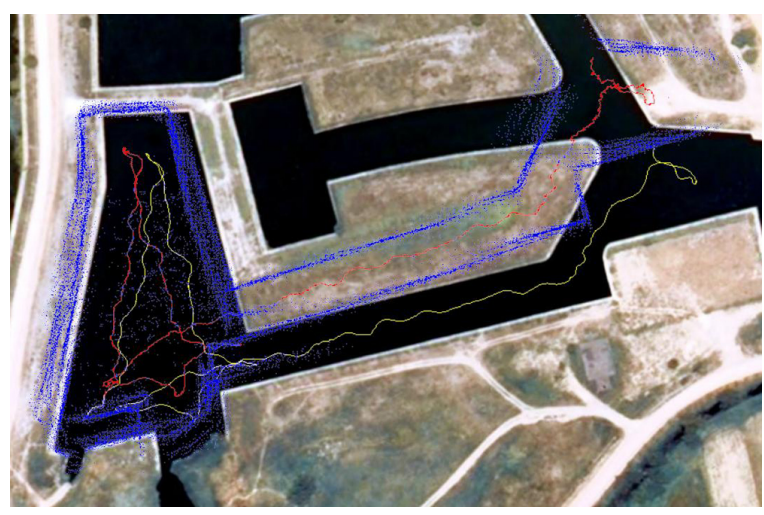

a)

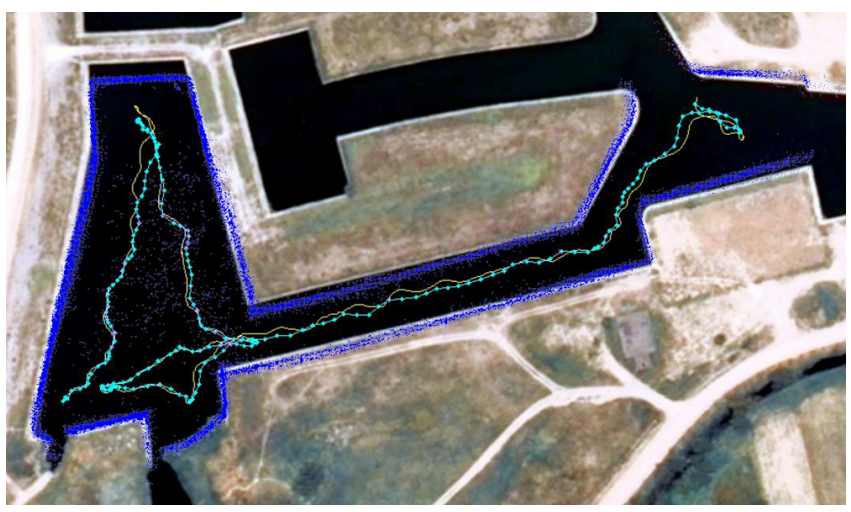

b)

Fig. 2. Results: a) Trajectory and map generated with odometry (red). GPS trajectory (yellow) used as a ground truth. b) Map and trajectory (dotted cyan) generated with the SLAM algorithm.

\section{CONCLUSIONS}

This paper proposes an extension to the MSISpIC algorithm in the pose-based SLAM framework. MSISpIC is able to perform underwater scan matching using a MSIS. To deal with the motion induced distortion of the acoustic image, an EKF is used to estimate the robot motion during the scan. The filter uses a constant velocity model with acceleration noise for motion prediction and velocity (DVL) and attitude measurements (MRU) for updating the state. Through the compounding of the relative robot position within the scan, with the range and bearing measurements of the beams gathered with the sonar, the acoustic image gets undistorted. Assuming Gaussian noise, the algorithm is able to predict the uncertainty of the sonar measurements with respect to a frame located at the position occupied by the robot at the end of the scan. Each full scan pose is maintained in a second filter, an augment EKF and is cross registered with all the previous scan posses that are in a certain range applying the spIC algorithm. The proposed method has been tested with a real world dataset including DGPS for ground truth acquired during a survey mission in an abandoned marina located in the Girona coast. The results show substantial improvements in trajectory correction and map reconstruction.

\section{FUTURE WORK}

Currently we are working on the calculation of the real uncertainty of the scan matching algorithm which will depend of the uncertainties of the input scans. Next step will consist of gathering a new dataset in an complete unstructured environment to check how the algorithm performs.

\section{ACKNOWLEDGMENTS}

This research was sponsored by the Marie Curie Research Training Network FREEsubNET (MRTN-CT-2006-036186) and by the Spanish government (DPI2005-09001-C03-01).

\section{REFERENCES}

[1] T. Bailey and H. F. Durrant-Whyte, "Simultaneous localization and mapping (slam): Part ii, state of the art," IEEE Robotics and Automation Magazine, vol. 13, no. 3, pp. 108-117, 2006.
[2] R. Eustice, H. Singh, J. Leonard, M.Walter, and B. R., "Visually navigating the rms titanic with slam information filters," in Proc. Robotics: Science and Systems, MIT Press, Cambridge, MA, pp. 57-64.

[3] C. WangThorpe and A. Suppe, "Ladar-based detection and tracking of moving objects from a ground vehicle at high speeds," Intelligent Vehicles Symposium, 2003. Proceedings. IEEE, pp. 416-421, 9-11 June 2003.

[4] D. Hahnel, W. Burgard, D. Fox, and S. Thrun, "An efficient fastslam algorithm for generating maps of large-scale cyclic environments from raw laser range measurements," Intelligent Robots and Systems, 2003. (IROS 2003). Proceedings. 2003 IEEE/RSJ International Conference on, vol. 1, pp. 206-211 vol.1, 27-31 Oct. 2003.

[5] A. Grossmann and M. Poli, "Robust mobile robot localisation from sparse and noisy proximity readings," 1999. [Online]. Available: citeseer.ist.psu.edu/article/gro99robust.html

[6] J. Castellanos, J. Tardós, and J. Neira, "Constraint-based mobile robot localization," Advanced Robotics and Intelligent Systems, IEE, 1996.

[7] D. Ribas, N. Palomer, P. Ridao, M. Carreras, and E. Hernandez, "Ictineu AUV wins the first SAUC-E competition," in IEEE International Conference on Robotics and Automation, Roma, Italy, apr 2007.

[8] P. J. Besl and N. D. McKay, "A method for registration of 3-d shapes," IEEE Trans. Pattern Anal. Mach. Intell., vol. 14, no. 2, 1992.

[9] S. Rusinkiewicz and M. Levoy, "Efficient variants of the icp algorithm," 3-D Digital Imaging and Modeling, 2001. Proceedings. Third International Conference on, pp. 145-152, 2001.

[10] L. Montesano, J. Mínguez, and L. Montano, "Probabilistic scan matching for motion estimation in unstructured environments," Intelligent Robots and Systems, 2005. (IROS 2005). 2005 IEEE/RSJ International Conference on, Aug. 2005.

[11] A. Burguera, Y. Gonzalez, and G. Oliver, "A probabilistic framework for sonar scan matching localization," International Journal of the RSJ, Advanced Robotics, 2008.

[12] E. Hernandez, P. Ridao, D. Ribas, and J. Batlle, "Msispic: A probabilistic scan matching algorithm using a mechanical scanned imaging sonar," Journal on Physical Agents (JoPhA), vol. 3, no. 1, pp. 3-12, 2009.

[13] Y. Bar-Shalom and T. Fortman, Tracking and Data Association. Academic Press, 1998.

[14] D. Ribas, J. Neira, P. Ridao, and J. Tardós, "Auv localization in structured underwater environments using an a priori map," in 7th IFAC Conference on Manoeuvring and Control of Marine Crafts, Lisbon, Portugal, september 2006.

[15] T. Fossen, Guidance and Control of Ocean Vehicles, J. Wiley and S. Ltd., Eds., 1994.

[16] D. Ribas, P. Ridao, J. Tardós, and J. Neira, "Underwater SLAM in man made structured environments," Journal of Field Robotics, vol. Accepted for publication, 2008.

[17] R. Smith, M. Self, and P. Cheeseman, "Estimating uncertain spatial relationships in robotics," in Autonomous robot vehicles. New York, NY, USA: Springer-Verlag New York, Inc., 1990, pp. 167-193.

[18] D. Ribas, "David ribas homepage," http://eia.udg.edu/ dribas, Online March 2009. 